\title{
Atom microscopy comes of age
}

\author{
Four years after the first demonstration that beams of atoms can interfere with each other just as if they were beams of \\ light, it has been possible to extract useful information from an experiment. But emulators will be few.
}

QUANTUM duality goes back a long way, to the beginnings of optical interferometers. Of course, in the mid-nineteenth century, there was no reason to believe (Newton's corpuscular theory of optics notwithstanding) that there were particles of radiation, or that they might have the properties of waves and of particles in different circumstances. People could work with the interferometers of the late nineteenth century in complete contentment that wave theories could account for everything.

Only Planck's version of the quantum theory rescued the notion that there may be quanta of radiation; in 1905, Einstein's theory of the photoelectric effect clinched the point. Proof of the quantum duality of electrons had to wait a further twenty years. Since then, people's ambitions have enormously expanded. Beams of neutrons are routinely used in studies of the atomic structures of solids, for they too have the properties of waves as well as of particles, with a wavelength $(\lambda)$ nicely given by the de Broglie specification of $h /(m v)$, where $h$ is Planck's constant and $m v$ the momentum of each particle.

So why not use other kinds of particle instead, thereby further extending the range of wavelengths over which interference or diffraction techniques can usefully be practised? There are some obvious snags. For one thing, charged particles will not do; imagine trying to make beams of slow-moving protons whose electric charges would not blow them apart! (But fast-moving protons may well have quantum wavelengths comparable with nuclear dimensions and so be probes of the internal structure of atomic nuclei - as well as of their own.) Another is that the electronic structure of an atom may interact with other objects in its path, perhaps making the collision inelastic.

None of that has deterred people from the construction of instruments dependent on the wave properties of neutral atoms. The first demonstrations that the matter-waves of atoms will indeed interfere with each other were independently provided just four years ago, by a group from the University of Konstanz (O. Carnal \& J. Mlynek Phys. Rev. Lett. 66, 2689-2692; 1991) and another from the Massachusetts Institute of Technology (MIT) (D. W. Keith, C. R. Ekstrom, Q. A. Turchette \& D.E. Pritchard Phys. Rev. Lett. 66, 2693-2696; 1991). The former used beams of metastable helium atoms, the latter went for sodium atoms instead.
The difficulty of these experiments is constructing the slits and gratings through which the atoms must pass, and which have dimensions measured in fractions of a micrometre.

A team of six grown from the MIT group (two of whom are also affiliated with the University of Innsbruck) has now neatly turned one of the snags of atombased interferometers - the uncertainties caused by collisions - to their advantage (J. Schmiedmayer et al. Phys. Rev. Lett. 74, $1043-1047 ; 1995)$. Since next to nothing is directly known of the low-energy collision of one atom with another, why not use an atom interferometer to measure them?

The basic instrument is an elaboration of that described four years ago. There is a nearly mono-energetic beam of sodium atoms, obtained by ejecting through a nozzle into a vacuum chamber sodium atoms carried by argon gas. From the estimated velocity of the gas through the nozzle, the de Broglie wavelength of the sodium atoms is 16 picometres, or 0.16 angstrom. The group uses a grating to split the beam into several sub-components, of which only two are needed. The gratings have a geometrical period of 200 $\mathrm{nm}$ (a fifth of a micrometre) and are fabricated as films of silicon nitride $\left(\mathrm{Si}_{3} \mathrm{~N}_{4}\right)$ using all the razzmatazz of silicon-chip technology.

In the original experiment, when the MIT group was simply concerned to demonstrate that interference happens, it operated in as good a vacuum as it could make. Even so, there were bizarre complications. Movement of the equipment as a whole, for example, creates a relativistically significant displacement of the measuring equipment relative to the sodium atoms in the beam, for which reason there is a system controlled by a laser driven servomechanism to correct for displacements of the equipment.

And the equivalent of the photographic film or the photomultipliers with which optical interference would be recorded? Simply a wire heated to the point at which sodium atoms will be ionized and a device for recording the arrival of electrons. Given the system for compensating for the chance movement of the equipment as a whole, it is easier to scan across the diffraction fringes by displacing the equipment than by moving the detector.

That was four years ago. How, in 1995, to cause one of two beams of sodium atoms to interact with the atoms of an ambient gas when sodium atoms, unlike neutrons, will not pass through the thinnest barriers? Schiedmayer et al. have devised a leaky gas chamber through which to pass one of the two beams of a double-beam interferometer. They have built a rectangular box, open at the ends, which is divided in two by a thin sheet of aluminized mylar foil. One half of the box is fitted with a nozzle through which gas can enter. Except for a gap $200 \mu \mathrm{m}$ across, the ends of that half of the box are closed by metal baffles.

So the complete experiment consists of three 200-nm diffraction gratings, one to split the beam, one to select the subbeams immediately in advance of the doubled interaction chamber and one immediately before the hot wire. The gases used to interact with one of the two beams are the noble gases (up to xenon) together with $\mathrm{N}_{2}, \mathrm{NH}_{3}, \mathrm{CO}_{2}$ and $\mathrm{H}_{2} \mathrm{O}$. The essential measurement is that of the phase shift between the two beams occasioned by the presence of scattering gas in one of the two arms. Direct measurement of the density of gas in the interacting cell is evidently not possible, especially because the supposedly empty half of the chamber must become contaminated, but the attenuation of the beams serves as a control on the amount of scattering material.

In reality, the MIT group has been able to finesse even that difficulty by persuading itself that its real interest lies in the ratio of the real and imaginary parts of the forward scattering amplitude — the chance that a sodium atom will be unaffected by an encounter with an ambient atom of the scattered gas. That is directly related to the refractive index of the gas concerned for matter waves of sodium (with a velocity of $1,000 \mathrm{~m} \mathrm{~s}^{-1}$ ).

For what it is worth, the results say something about the interaction of sodium atoms with the rare gases. There is an attrractive potential-well for each of them, but it is predictably deepest for xenon and decreases steadily from there down to helium. Xenon interacts with sodium as if by a van der Waals interaction, but helium may just as well behave like a hard-sphere billiard ball. The authors say that, apart from improving the sensitivity of their measurements, they hope to extract further information about these scattering processes by varying the energy (and de Broglie wavelength) of their sodium atoms. Good luck to them! will be the general reaction.

John Maddox 